\title{
A Social Program for the Early Detection of Pancreatic Cancer: The Kishiwada Katsuragi Project
}

\author{
Hiroki Sakamoto ${ }^{a}$ Satoshi Harada ${ }^{a}$ Nobu Nishioka ${ }^{a}$ Kazuo Maeda ${ }^{b}$ \\ Takamasa Kuriharac $^{c}$ Tateki Sakamoto $^{d}$ Kazuhide Higuchi $^{\mathrm{e}}$ Masayuki Kitano $^{f}$ \\ Yoshifumi Takeyamag Masafumi Kogire ${ }^{\mathrm{h}}$ Masatoshi Kudo $^{\mathrm{i}}$ \\ ${ }^{a}$ Department of Gastroenterology, Katsuragi Hospital, ${ }^{\mathrm{b}}$ Internal Medicine, Maeda Clinic, ${ }^{\mathrm{c} I n t e r n a l}$ Medicine, Kurihara \\ Clinic, ${ }^{d}$ Internal Medicine and Pediatrics, Sakamoto Clinic, and ${ }^{e}$ 2nd Department of Internal Medicine, Osaka

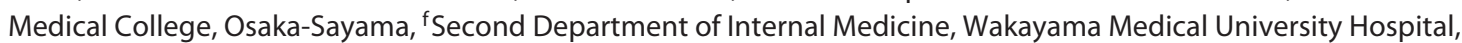 \\ Wakayama, and Departments of Surgery at ${ }^{9}$ Kindai University Faculty of Medicine and h Kishiwada City Hospital, and \\ 'Department of Gastroenterology and Hepatology, Kindai University Hospital, Osaka-Sayama, Japan
}

\section{Keywords}

Pancreatic cancer · Early pancreatic cancer · Intraductal papillary mucinous neoplasm · Endoscopic ultrasound . Kishiwada Katsuragi project

\begin{abstract}
Objectives: The early-stage pancreatic cancer (e-PC; stage I/ II) detection rate is quite low at approximately $25 \%$. The aim of this study was to evaluate the feasibility of a social program (the Kishiwada Katsuragi project) wherein our hospital, which specializes in $P C$, and primary care medical offices (PMOs) used clinical findings to detect e-PC. Methods: Patients with a score of $\geq 2$ points on clinical findings were enrolled: symptoms of abdominal pain/back pain (1 point), new-onset diabetes (1 point), high amylase (AMY) and/or pancreaitc AMY (P-AMY) (1 point), high carbohydrate antigen 19-9 (1 point), and ultrasonography (US) findings including direct (e.g., a solid pancreatic tumor) and/or indirect findings (e.g., dilatation of a pancreatic diameter of $\geq 2.5 \mathrm{~mm}$
\end{abstract}

\section{KARGER}

(C) 2017 S. Karger AG, Basel

E-Mail karger@karger.com

www.karger.com/ocl and/or cystic lesions) (2 points) were evaluated using the protocol for social programs. Results: Between November 2014 and December 2016, 244 patients were enrolled by 41 PMOs as cooperative facilities, and 15 e-PC cases (53.6\%) of the 28 PC patients were detected. The mean clinical finding score of the e-PC group $(3.13 \pm 1.9)$ was significantly higher than that of the overall non-PC group $(2.1 \pm 0.4)(p<0.05)$. "High AMY/P-AMY" and "symptoms" were significantly more frequent in the e-PC group than in the non-PC group ( $p<$ $0.05)$. Although the sensitivity of direct findings by US was $40.0 \%$, that of indirect-findings was $93.3 \%$ in the e-PC group. Nine and 6 of the 15 patients with e-PC were enrolled via general internal medicine offices (GIMs) and other PMOs without GIMs (general surgery, $n=3$; urology, $n=2$; otolaryngology, $n=1$ ). Conclusion: This social program with collaborations between medical centers that specialize in PC and PMOs used clinical findings, suggesting that not only GIMs but also other PMOs and indirect findings by US may play an important role in improving the e-PC detection rate.

(c) 2017 S. Karger AG, Basel

Dr. Hiroki Sakamoto

Department of Gastroenterology

Katsuragi Hospital

22-33-1 Habu-cho, Kishiwada, Osaka 596-0825 (Japan)

E-Mail hiroki.sakamoto@ nifty.com 


\section{Introduction}

Pancreatic cancer (PC) is generally associated with a poor prognosis and is difficult to diagnose early enough for surgical resection, the only potentially curative treatment for PC, to be performed. Indeed, only $25 \%$ of PC patients have early-stage PC (e-PC) at the time of detection $[1,2]$.

A screening program is needed to improve the detection rate of e-PC. Previous studies have identified various risk groups and factors (a family history of PC and pancreatitis, smoking, alcohol consumption, diabetes (DM), obesity, and cystic lesions, including intraductal papillary mucinous neoplasm and main pancreatic duct [MPD] dilatation), but few standard programs are available for screening high-risk patients. Evidence-based clinical guidelines for PC were published by the Japan Pancreas Society (JPS) in 2006, with the third edition released in 2013 [3]. The guidelines include a protocol for diagnosing and treating PC. The sections of Clinical questions 1 and 2 suggest several risk factors that may be involved in the development of PC [4-11].

The guidelines include a protocol for a social program to diagnose e-PC by consulting clinical findings (the
Kishiwada Katsuragi project). The aim of the present study was to evaluate the feasibility of such a social program via collaboration between our medical center, which specializes in PC, and primary-care medical offices (PMOs).

\section{Patients and Methods}

Forty-one PMOs, including 27 general internal medicine offices (GIMs) and 14 other PMOs (otolaryngology, $n=4$; general surgery, $n=3$; orthopedic surgery, $n=2$; gynecology, $n=2$; urology, $n=2$, and psychiatry, $n=1$ ), were enrolled. If a patient presented with $\geq 2$ points for 5 clinical findings, they were examined in accordance with the protocol for social programs for diagnosing e-PC (Fig. 1). If a patient did not receive ultrasonography (US) and/or did not undergo a carbohydrate antigen 19-9 (CA199) examination by the PMO at entry into this study, then they received these evaluations before undergoing magnetic resonance cholangiopancreatography (MRCP) including magnetic resonance imaging (MRI), and endoscopic ultrasound (EUS) at our institution.

Between November 2014 and October 2016, 244 patients in whom PC was suspected based on the clinical findings (at the cooperating facilities) were evaluated. Twenty-eight (11.5\%) of these 244 patients were ultimately diagnosed with PC. This study received approval from the ethics committee of Katsuragi Hospital.
Fig. 1. The protocol for the social program for diagnosing early-stage pancreatic cancer. ENPD, endoscopic nasopancreatic drainage; ERCP, endoscopic retrograde cholangiopancreatography; EUS, endoscopic ultrasonography; FNA, fine-needle aspiration; MPD, main pancreatic duct; MRCP, magnetic resonance cholangiopancreatography; MRI, magnetic resonance imaging; US, ultrasonography; CA19-9, carbohydrate antigen 19-9; P-AMY, pancreatic amylase.

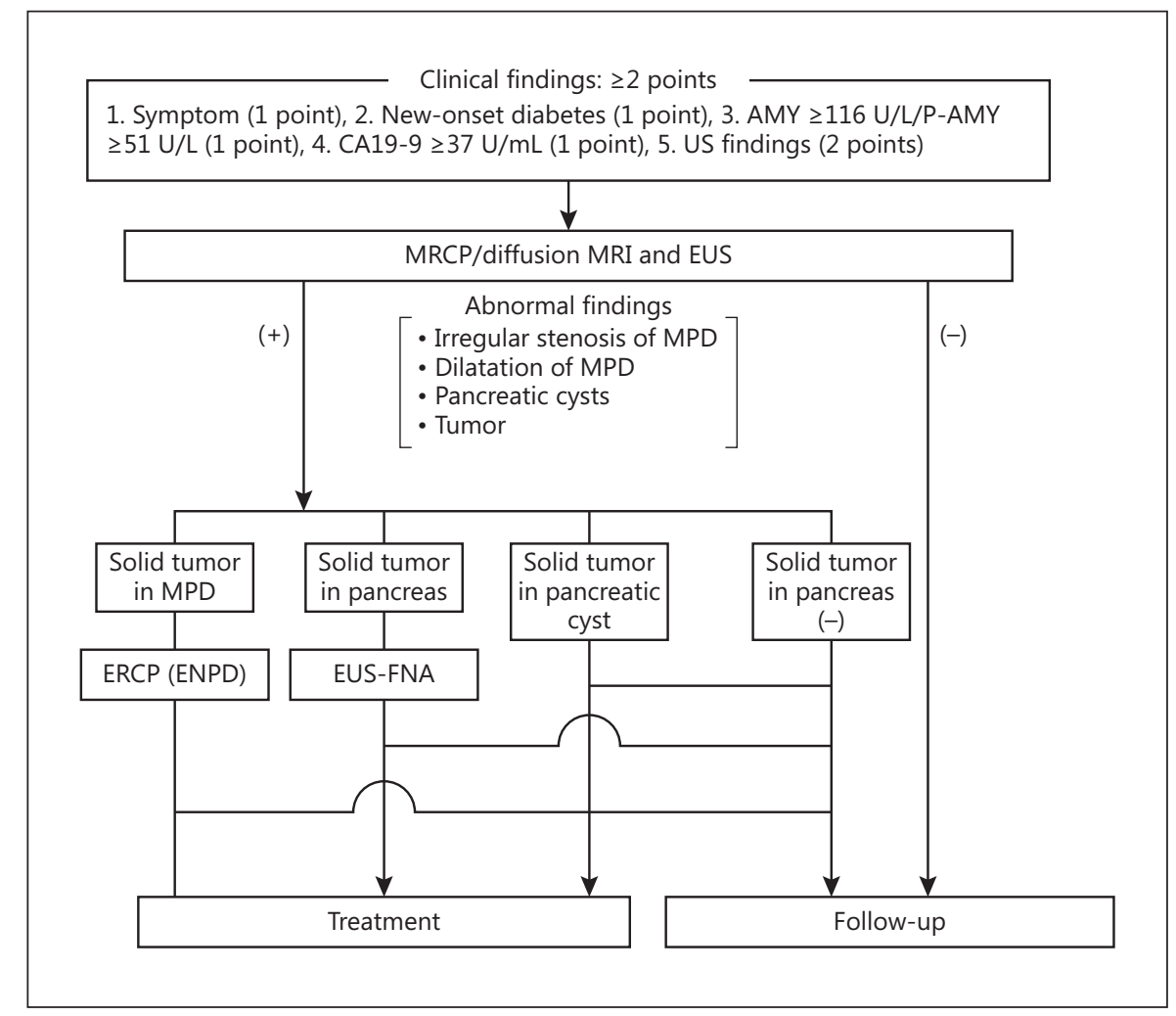

Sakamoto et al. 


\section{Clinical Findings}

Patients with 2 points for the following clinical findings were diagnosed with suspected PC: (1) symptoms (abdominal pain or fatigue, or back pain) (1 point) [3], (2) new-onset DM (1 point) [12-14], (3) a high amylase (AMY) level ( $\geq 116 \mathrm{U} / \mathrm{L})$ and/or pancreatic amylase (P-AMY) level ( $\geq 51 \mathrm{U} / \mathrm{L})$ (1 point) [3], (4) a high CA19-9 level ( $\geq 37 \mathrm{U} / \mathrm{mL}$ ) (1 point) [15-17], and (5) US findings (2 points) $[18,19]$. The US findings included direct (e.g., a solid pancreatic tumor) and indirect findings (e.g., dilatation of the MPD to a diameter of $\geq 2.5 \mathrm{~mm}$ or a pancreatic cyst). DM was defined as a fasting blood sugar level of $\geq 126 \mathrm{mg} / \mathrm{dL}$, a casual blood glucose level of $\geq 200 \mathrm{mg} / \mathrm{dL}$, or HbAlc (NGSP) $\geq 6.5 \%$.
Protocol for Social Programs for Diagnosing e-PC (Fig. 1)

The JPS guidelines include a protocol for diagnosing and treating PC [3]. The protocol in the present study (the Kishiwada project) referenced the protocol for the diagnosis of PC.

Patients who were enrolled based on their clinical findings underwent diffusion-weighted imaging (DWI) MRI and MRCP, and EUS (GF-UC240P-AL5; Olympus, Tokyo, Japan) was performed. If EUS revealed a solid mass in the pancreas, then EUS-fine needle aspiration (EUS-FNA) was performed $[20,21]$. If the findings revealed irregular stenosis in the MPD or dilatation of the MPD, then endoscopic retrograde cholangiopancreatography was performed, and a cytological examination of pancreatic juice samples obtained by multiple endoscopic nasopancreatic drainage (ENPD) samplings was performed (Fig. 1).

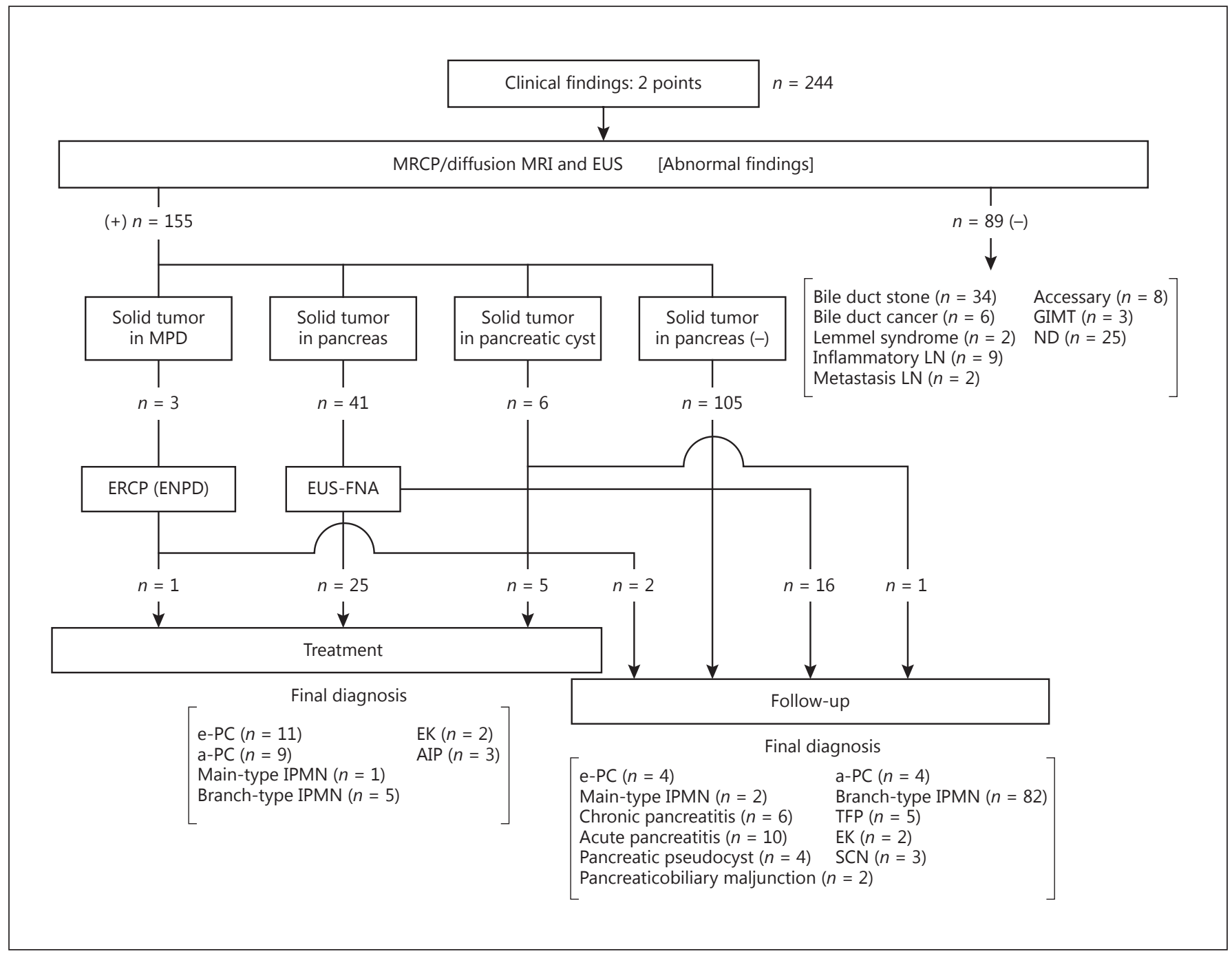

Fig. 2. Clinical characteristics of patients. a-PC, advanced pancreatic cancer; e-PC, early-stage pancreatic cancer; EK, neuroendocrine; LN, lymph node; AIP, autoimmune pancreatitis; TFP, tumor-forming pancreatitis; GIMT, gastrointestinal mesenchymal tumor; SCN, serous cyst adenoma; IPMN, intraductal papillary mucinous neoplasm; ND, no disease. For all other abbreviations, see Figure 1.

A Social Program for the Early Detection of Pancreatic Cancer
Oncology 2017;93(suppl 1):89-97 DOI: $10.1159 / 000481239$ 

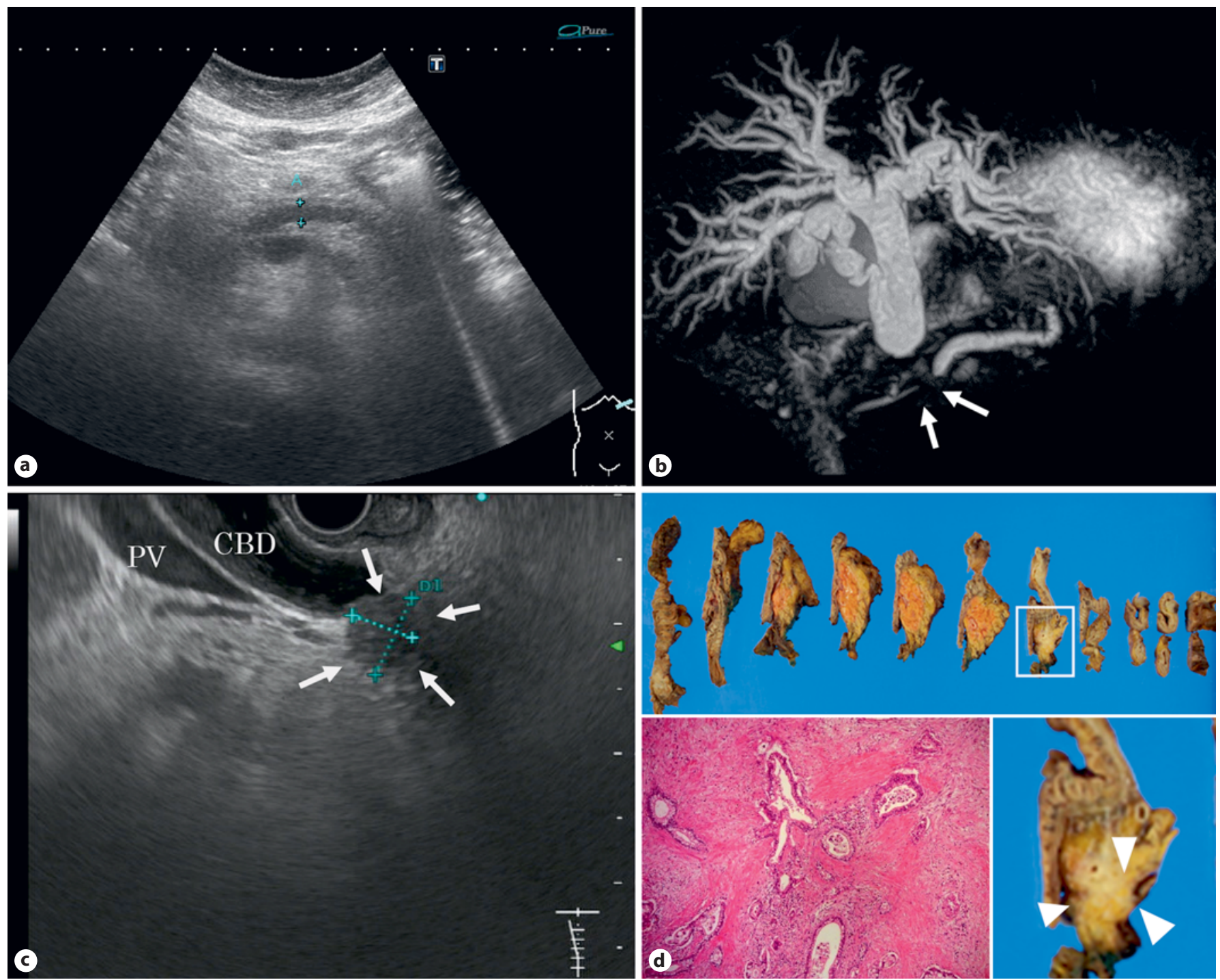

Fig. 3. A 66-year-old woman positive at entry for 3 points ("symptoms [abdominal pain]" and "US findings") of 5 clinical findings from a primary-care medical office. a Ultrasonography did not detect a tumor without dilatation of the main pancreatic duct. b Magnetic resonance cholangiopancreatography shows stenosis and dilatation of the main pancreatic duct (arrow). c Endoscopic ultrasonography shows a low-echoic lesion measuring $18 \mathrm{~mm}$ in diameter (arrow) adjacent to the bile duct. The histological diag-

\section{DWI-MRI and MRCP Techniques}

MRI examinations were carried out with a 2.0 -Tesla unit (Vantage Titan, Toshiba, Japan) with a 12-channel body and spine matrix coil combination. DWI was performed as a part of the routine abdomen MRI protocol for the evaluation of hepato-pancreato-biliary ducts in our institution. The protocol included axial fat-sat T1-weighted images, axial T2-weighted images, axial T2weighted half-Fourier acquisition single-shot turbo spin echo images, coronal 2D MRCP, coronal 3D MRCP, and DWI images. nosis was adenocarcinoma by endoscopic ultrasonography-fine needle aspiration. d She received subtotal stomach-preserving pancreatoduodenectomy. The resected specimen showed a white solid tumor, measuring $20 \times 18 \mathrm{~mm}$ (arrowheads). A pathologic examination of the resected specimen showed invasive ductal carcinoma, moderately differentiated. The final pathological diagnosis was stage IIA pancreatic cancer. PV, portal vein; CBD, common bile duct.

Apparent diffusion coefficient maps were automatically reconstructed for all DWI.

\section{Diagnosis and Classification of PC}

The final diagnoses were made according to the histology of the resected tumor. For patients who did not undergo surgical resection, the final diagnoses were made according to the histology or cytology of samples obtained by EUS-FNA or ENPD. In cases where pancreatic malignancy was excluded by EUS-FNA or 
ENPD, the final diagnosis was confirmed by following the patient for at least 6 months.

Tumor classification was performed accordance with the Union for International Cancer Control (UICC) guidelines for PC. In this study, e-PC was defined as stage 0 -IIB and advanced PC (aPC) was defined as stage III-IV accordance with the UICC guidelines.

\section{Statistical Analyses}

The mean total scores of the e-PC, a-PC, and non-PC groups were compared using Wilcoxon's signed-rank test. The $\chi^{2}$ test was used to compare the frequencies of each clinical findings category between the e-PC, a-PC, and non-PC groups. Sensitivities for detecting pancreatic findings (direct and indirect) by MRCP were calculated and compared between the e-PC and a-PC groups. A $p$ value $<0.05$ was considered to be statistically significant.

\section{Results}

Among the 244 cases diagnosed with PC based on the clinical findings, pancreatic disease was detected in 155 cases $(63.5 \%)$, while diseases other than pancreatic disease were detected in $64(26.2 \%)$, and 25 cases (10.3\%) were found to have no disease at all (Fig. 2).

Three of these 155 patients received a cytodiagnosis using pancreatic juice obtained by ENPD, as EUS revealed a tumor in the MPD; they were therefore diagnosed with adenocarcinoma by cytology of the ENPD sample. One of these 3 patients underwent distal pancreatectomy, and the other 2 received best supportive care because they were elderly. Forty-one cases in whom EUS revealed a tumor in the pancreas parenchyma underwent EUS-FNA. The final UICC tumor classification of pancreatic adenocarcinoma revealed that 15 of the $28 \mathrm{PC}(53.6 \%)$ cases were e-PC (stage IA, $n=4$; IB, $n=1$; IIA, $n=4$; IIB, $n=6$ ); 11 and 4 patients in the e-PC group received surgery and best supportive care because 4 patients were elderly, respectively (Fig. 3). Thirteen of the 28 PC (46.4\%) cases were a-PC (stage III, $n=4$; IV, $n=9$ ); 9 and 4 patients in the a-PC group received chemotherapy and best supportive care, respectively. In 105 cases, EUS revealed a cystic lesion or pancreatic swelling but did not reveal a solid tumor in the pancreas; therefore, these patients are being followed up without EUS-FNA or ENPD (Fig. 2).

Comparison of the Mean Total Scores at Entry among the e-PC, $a-P C$, and Non-PC Groups (Fig. 4)

The average total scores were $3.1 \pm 1.9,3.9 \pm 0.9$, and $2.1 \pm 0.4$ in the e-PC, a-PC, and non-PC groups, respectively. The average total score of the e-PC and a-PC group

A Social Program for the Early Detection of Pancreatic Cancer

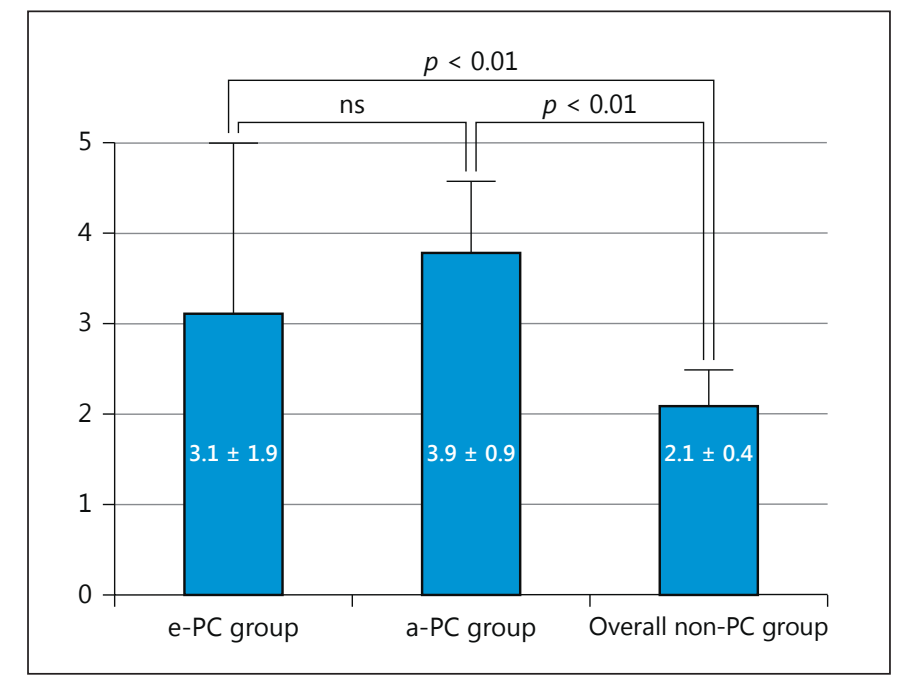

Fig. 4. A comparison of the mean total scores at entry among the early-stage pancreatic cancer (e-PC), advanced pancreatic cancer (a-PC), and non-pancreatic cancer (non-PC) groups. ns, not significant.

was significantly higher than that of the non-PC group $(p<0.05)$. There were no significant differences in the average total score between the e-PC and a-PC groups (Fig. 4).

Comparison of Each Score Item among the e-PC, a-PC, and Non-PC Groups (Fig. 5)

"High AMY/P-AMY" was found in 8 of 15 (53.3\%), 2 of $13(15.4 \%)$, and 36 of $216(16.7 \%)$ patients in the e-PC, a-PC, and non-PC groups, respectively; the frequency in the e-PC group was significantly higher than in the other groups $(p<0.05)$. "Symptoms" was found in 8 of 15 (53.3\%), 10 of $13(77 \%)$, and 42 of $216(19.4 \%)$ patients in the e-PC, a-PC, and non-PC groups, respectively; the frequency in the e-PC and a-PC groups was significantly higher than in the non-PC group $(p<0.05)$. "US findings" was found in 11 of 15 (73.3\%), 10 of 13 (66\%), and 160 of $216(74.5 \%)$ patients in the e-PC, a-PC, and non-PC groups, respectively, which was not significantly different. "New-onset DM" was found in 1 of 15 (6.7\%), 5 of 13 (38.5\%), and 17 of 216 (7.9\%) patients in the e-PC, a-PC, and non-PC groups, respectively; the frequency in the e-PC group was significantly higher than in the other groups $(p<0.05)$. "High CA19-9 levels" was found in 8 of $15(53.3 \%), 13$ of $13(100 \%)$, and 17 of $216(7.9 \%)$ patients in the e-PC, a-PC, and non-PC groups, respectively; the frequency in the e-PC group was significantly higher than in the non-PC group $(p<0.05)$.

Oncology 2017;93(suppl 1):89-97 93 


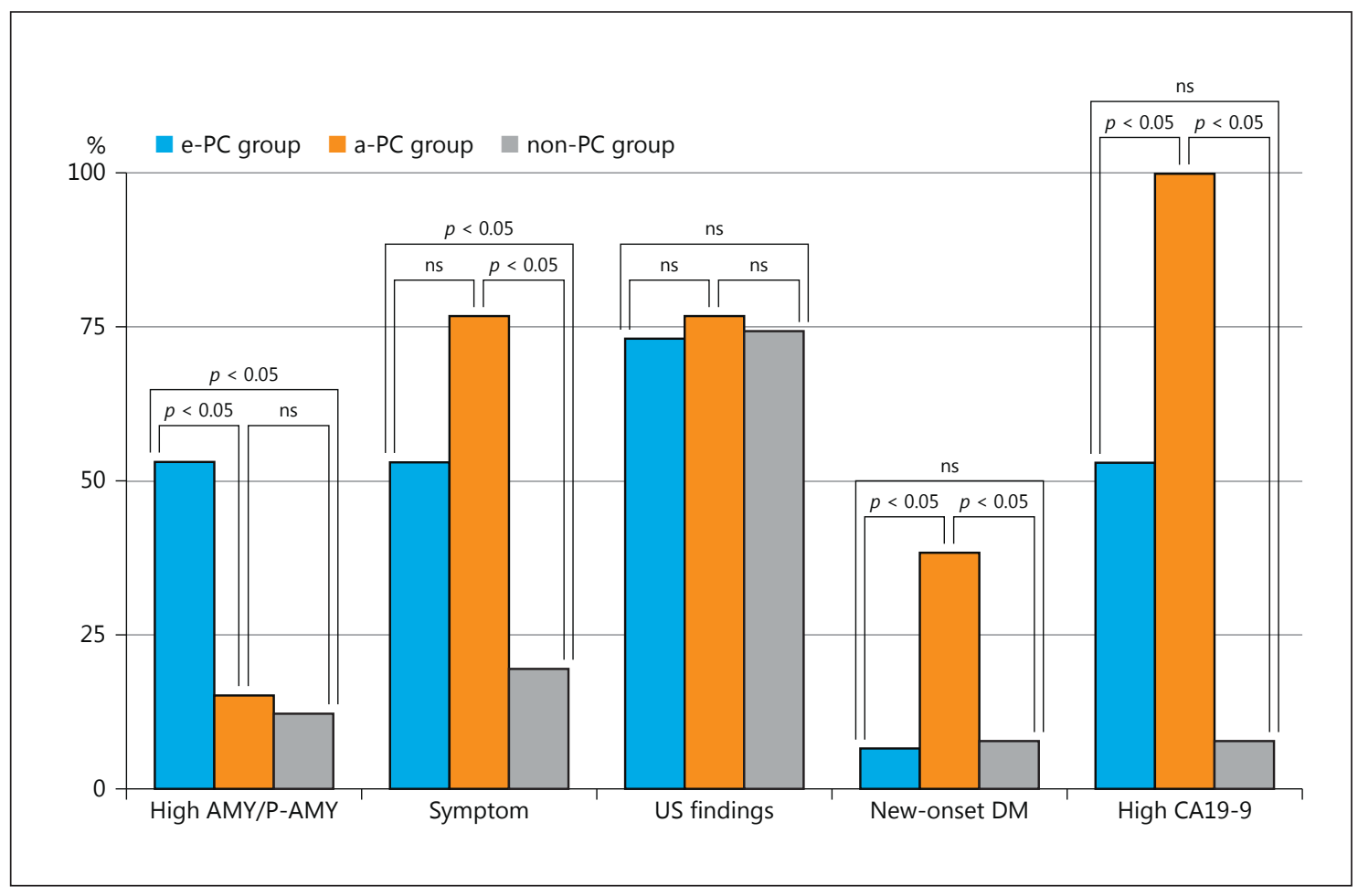

Fig. 5. A comparison of each score item among the early-stage pancreatic cancer (e-PC), advanced pancreatic cancer (a-PC), and non-pancreatic cancer (non-PC) groups. P-AMY, pancreatic amylase; US, ultrasonography; DM, diabetes mellitus; CA19-9, carbohydrate antigen 19-9; ns, not significant.

\section{Clinical Findings of the PC Patients at Entry}

Nine of the $15(60.0 \%)$ patients in the e-PC group, were enrolled via GIMs, while the remaining 6 (40.0\%) were enrolled via PMOs (general surgery, $n=3$; urology, $n=2$; otolaryngology, $n=1)$. Six patients with abdominal pain in the e-PC group had acute pancreatitis; the abdominal pain coincided with a high AMY level. Abnormal US findings were detected at entry in 11 of the 15 (73.3\%) patients in the e-PC group, and the remaining 4 (26.7\%) were enrolled via facilities in which US was not conducted. After having being introduced to our hospital, all of these patients underwent US. The total sensitivity of direct detection by US (both US findings at entry and at our institution) was $40.0 \%$ (6 of 15) in the e-PC group. The total sensitivity for detecting MPD dilatation by US was $66.7 \%$ ( 10 of 15 ) in the e-PC group. The sensitivity for detecting a cystic lesion by US was $20.0 \%$ ( 3 of $15)$ in the e-PC group. The overall sensitivity of indirect US findings (MPD dilatation and/or cystic lesion) by US was $93.3 \%$.

\section{Comparison of the Sensitivities of Detecting}

Pancreatic Findings by MRCP between the e-PC and a-PC Groups (Table 1)

The sensitivities for detecting pancreatic carcinoma by diffusion MRI were $33.3 \%$ (5 of 15) and 92.3\% (12 of 13) in the e-PC and a-PC groups, respectively, which was significantly different $(p<0.05)$. The sensitivities for detecting MPD dilatation by MRCP were 93.3\% (14 of 15) and $84.6 \%$ (11 of 13 ) in the e-PC and a-PC groups, respectively. The sensitivities for detecting MPD cystic lesion by MRCP were $26.7 \%$ (4 of 15 ) and $38.5 \%$ (5 of 13 ) in the e-PC and a-PC groups, respectively. There were no significant differences in the indirect findings for MPD dilatation and MPD cystic lesions between the e-PC and a-PC groups.

\section{Discussion}

PC is one of most lethal malignant cancers, ranking fifth in mortality related to cancer worldwide. The overall 5-year survival rate of PC has been reported to be 
Table 1. Total sensitivities for detecting pancreatic findings by diffusion magnetic resonance imaging (MRI) and magnetic resonance cholangiopancreatography (MRCP) between the early-stage pancreatic cancer (e-PC) and advanced pancreatic cancer (a-PC) groups

\begin{tabular}{|c|c|c|c|c|}
\hline & \multirow{2}{*}{$\begin{array}{l}\text { Direct finding on } \\
\text { diffusion MRI, } n(\%) \\
\text { solid tumor }\end{array}$} & \multicolumn{3}{|c|}{ Indirect findings on MRCP, $n(\%)$} \\
\hline & & MPD dilatation & cystic lesion & $\begin{array}{l}\text { MPD dilatation and/ } \\
\text { or cystic lesions }\end{array}$ \\
\hline $\begin{array}{l}\text { e-PC group }(n=15) \\
\text { a-PC group }(n=13)\end{array}$ & $\left.\begin{array}{r}5(33.3) \\
12(92.3)\end{array}\right\} p<0.05$ & $\left.\begin{array}{l}14(92.3) \\
11(84.6)\end{array}\right\} \mathrm{ns}$ & $\left.\begin{array}{l}4(26.7) \\
5(38.5)\end{array}\right\} \mathrm{ns}$ & $\left.\begin{array}{l}8(100) \\
6(100)\end{array}\right\} \mathrm{ns}$ \\
\hline
\end{tabular}

MPD, main pancreatic duct; ns, not significant.

around 1-4\% [22]. Egawa et al. [23] analyzed 32,619 cumulative records from 1981 to 2007 . The survival curve in their report was divided into that for patients who underwent pancreatectomy and that for those who had unresectable disease. There was a significant increase in the survival rate in the patients who underwent resection. Chiang et al. [24] compared the clinicopathological characteristics and prognosis of large PC (tumor size $>2 \mathrm{~cm}$ ) and small PC (tumor size $\leq 2 \mathrm{~cm}$ ) patients undergoing resection. Small-PC and large-PC patients had a similar prognosis after resection [23]. A high albumin level ( $>3.5$ $\mathrm{g} / \mathrm{dL}$ ) and early-stage disease (stage I, II) were favorable prognosis factors for small-PC patients. The detection of PC at an early stage and the performance of curative surgery can be expected to improve the long-term patient outcomes.

Hanada et al. $[4,22]$ reported that a social program for the early diagnosis of PC, with collaborations between Onomichi Hospital, specialists in PC from medical centers, and general practitioners (Onomichi project), was useful for the early detection of PC. Developing an effective screening strategy for PC will require the establishment of a network wherein facilities specializing in PC cooperate with medical offices of various channels. Screening for cases of PC is simple and easy for various doctors, and a sensitive screening method that is safe with low invasiveness eagerly waited.

The pathogenesis of neoplasm remains unclear, but some risk factors have been noted for identifying candidates for screening, such as DM, obesity, cigarette smoking, a family history of PC, chronic pancreatitis, and intraductal papillary mucinous neoplasm. Evidence-based clinical guidelines for PC were published by the Japan Pancreas Society (JPS) in 2013 [3]. The sections of Clinical questions 1 and 2 suggest some risk factors that may

A Social Program for the Early Detection of Pancreatic Cancer be involved in the development of PC [4-11]. With reference to this information, this social program (Kishidawa Katsuragi project) adopted clinical findings.

We compared each item in the scores among the e-PC, a-PC, and non-PC groups. "US findings" showed no significant difference among the three groups. Three of the five clinical findings ("symptoms," "new onset DM," and "high CA19-9") were more frequent in the a-PC group than in the other groups. In contrast, "high AMY/PAMY" was significantly more frequent in the e-PC group than in the other groups. Six patients with abdominal pain in the e-PC group had acute pancreatitis; abdominal pain coincided with a high AMY/P-AMY level. Thus, regarding the clinical findings, abdominal pain in patients with a high AMY/P-AMY level was important for the detection of e-PC. Homma et al. [25] reported that, in a study of 10,162 asymptomatic individuals, abnormal CA19-9 levels were only noted in $18(0.2 \%)$ subjects. Although their study used a variety of screening tests, only 4 cases of PC $(0.04 \%)$ were detected [25]. The measurement of CA19-9 levels alone was therefore ineffective as a screening test for PC, especially in asymptomatic individuals. In our study, when the finding of a high CA19-9 level was combined with at least one other clinical finding, the rate of CA19-9 elevation in the e-PC group at entry was $53.3 \%$. As such, the combined finding of a high CA19-9 level and another clinical finding may be useful for detecting e-PC.

In this study, the total (both US findings at entry and our institution) sensitivity for detecting a direct finding by US was $40.0 \%$ in the e-PC group. However, the sensitivities for indirect findings, such as MPD dilatation or cystic lesions, by US were high and were therefore deemed very important for the detection of e-PC. Although the diagnostic ability of US depends on the operator's experi-

Oncology 2017;93(suppl 1):89-97 DOI: $10.1159 / 000481239$ 
ence and the patient's condition (in terms of obesity and bowel gas content), US remains useful noninvasive, convenient technique, and it is associated with lower costs than multiple detector computed tomography (MDCT), $\mathrm{MRCP} /$ diffusion MRI, and EUS, which are necessary primary imaging modalities for diagnosing e-PC.

Although there were no significant differences in the average total score between the e-PC and a-PC groups, the average total scores of the e-PC and a-PC groups were significantly higher than those of the overall non-PC groups at entry. Therefore, our findings suggested that if the average score for clinical findings was $\geq 3$, we should strongly doubt pancreatic cancer and perform a more detailed inspection with MRCP/diffusion MRI, EUS and MDCT.

In the Kishiwada Katsuragi project, patients who were enrolled according to clinical findings initially underwent $\mathrm{MRCP} /$ diffusion MRI. MDCT involves radiation exposure and carries an unpredictable risk of an allergic response to the iodine contrast medium; however, MRCP/ diffusion MRI carries no such risk or exposure requirements. In addition, MRCP is a noninvasive and increasingly common procedure in cases of biliary and pancreatic diseases and is as sensitive as endoscopic retrograde cholangiopancreatography in detecting pancreatic carcinomas [26-28]. Furthermore, diffusion MRI measures the Brownian motion (random thermal diffusion) of molecules in tissues and depicts locoregional characteristics of water diffusion. Thus, diffusion MRI can be used to distinguish neoplastic lesions from the surrounding normal tissues without the need for an imaging contrast agent. Diffusion MRI can be used for the diagnosis of PC. In our study, patients who were enrolled based on their clinical findings initially underwent diffusion MRI/ MRCP. In our study, the sensitivities of detecting direct finding by diffusion MRI were 33.3 and $92.3 \%$ in the e-PC and a-PC groups, respectively. Although diffusion MRI was useful for detecting a-PC, it was not sufficient to detect e-PC. However, in the e-PC group, the sensitivities for overall indirect findings, including dilatation of the
MPD and/or cysts, was $100 \%$, suggesting that these findings were very important for detecting e-PC.

In the Kishiwada Katsuragi project, we hoped that the clinical findings would allow e-PC to be readily detected by any PMO. Therefore, 41 PMOs (27 GIMs and 14 other PMOs) were enrolled as cooperative facilities, including 27 GIMs and 14 other types of PMO (general surgery, urology, and orthopedics departments) to determine which clinical findings were most closely associated with the detection of PC. Six (6/15) of all patients with e-PC were detected by other PMOs, suggesting that the participation of other PMOs, as well as GMIs, plays an important role in improving the e-PC detection rate. Regarding the clinical findings at entry, the rates of patients with symptoms, high AMY/P-AMY, high CA19-9, and new-onset DM were lower than those with US findings in the e-PC groups; however, these findings were important for the detection of PC because 4 of the 15 (26.7\%) patients were from facilities in which US was not conducted.

The present study is associated with several limitations, including the small study population. Further examinations are therefore necessary. The accumulation of a greater number of cases and the modification of the clinical finding score will be necessary to improve the efficiency of PC detection.

In conclusion, social programs with collaborations between medical centers that specialize in PC and PMOs used clinical findings, suggesting that not only GIMs but also other PMOs may be important for the early detection of PC. Findings from US and MRCP/diffusion MRI, especially indirect findings such as dilatation of the MPD and cystic lesions, were useful for the detection of e-PC. Further studies with the accumulation of a greater number of cases are necessary to confirm our findings.

\section{Disclosure Statement}

There are no companies in a relation of conflict of interest requiring disclosure in relation to this paper.
References

96
1 Egawa S,Toma H, Ohigashi H, Okusaka T, Nakao A, Hatori T, Maguchi H, Yanagisawa A, Tanaka M: Japan Pancreatic Cancer Registry; 30th year anniversary: Japan Pancreas Society. Pancreas 2012;41:985-992.

2 D'Haese JG, Werner J: Resectability of pancreatic cancer: new criteria. Radiologe 2016; $56: 318-324$.
3 Yamaguchi K, Okusaka T, Shimizu K, Furuse J, Ito Y, Hanada K, Shimosegawa T: Committee for revision of clinical guidelines for pancreatic cancer of Japan Pancreas Society. EBM-based clinical guidelines for pancreatic cancer (2013) issued by the Japan Pancreatic Society: a synopsis. Jpn J Clin Oncol 2014;44: 883-888.
6

(2)

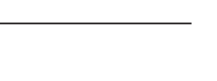

Oncology 2017;93(suppl 1):89-97

DOI: $10.1159 / 000481239$
Sakamoto et al. 
4 Hanada K, Okazaki A, Hirano N, Izumi Y, Teraoka Y, Ikemoto J, Kanemitsu K, Hino F, Fukuda T, Yonehara S: Diagnostic strategies for early pancreatic cancer. J Gastroenterol 2015; 50:147-154.

5 Okano K, Suzuki Y: Strategies for early detection of resectable pancreatic cancer. World J Gastroenterol 2014;20:11230-11240.

6 Raimondi S, Lowenfels AB, Morselli-Labate AM, Maisonneuve P, Pezzilli R: Pancreatic cancer in chronic pancreatitis; aetiology, incidence and early detection. Best Prac Res Clin Gastroenterol 2010;24:349-358.

7 McKay CJ, Glen P, McMillan DC: Chronic inflammation and pancreatic cancer. Best Pract Res Clin Gastroenterol 2008;22:65-73.

8 Rebours V, Boutron-Ruault MC, Schnee M, Férec C, Maire F, Hammel P, Ruszniewski P, Lévy P: Risk of pancreatic adenocarcinoma in patients with hereditary pancreatitis: a national exhaustive series. Am J Gastroenterol 2008;103:111-119.

9 Klein AP, Brune KA, Petersen GM, Goggins M, Tersmette AC, Offerhaus GJ, Griffin C, Cameron JL, Yeo CJ, Kern S, Hruban RH: Prospective risk of pancreatic cancer in familial pancreatic cancer kindreds. Cancer Res 2004;64:2634-2638.

10 Canto MI, Harinck F, Hruban RH, Offerhaus GJ, Poley JW, Kamel I, Nio Y, Schulick RS, Bassi C, Kluijt I, Levy MJ, Chak A, Fockens P, Goggins M, Bruno M: International Cancer of Pancreas Screening (CAPS) Consortium. International Cancer of the Pancreas Screening (CAPS) consortium summit on the management of patients with increased risk for familial pancreatic cancer. Gut 2013;62:339-347.

11 Brune KA, Lau B, Palmisano E, Canto M, Goggins MG, Hruban RH, Klein AP: Importance of age of onset in pancreatic cancer kindreds. J Nat Cancer Inst 2010;102:119-126.

12 Pannala R, Basu A, Petersen GM, Chari ST: New-onset diabetes: a potential clue to the early diagnosis of pancreatic cancer. Lancet Oncol 2009;10:88-95.

13 Ogawa Y, Tanaka M, Inoue K, Yamaguchi K, Chijiiwa K, Mizumoto K, Tsutsu N, Nakamura YA: A prospective pancreatographyic study of the prevalence of pancreatic carcinoma in patients with diabetes mellitus. Cancer 2002;94:2344-2349.

14 Mizuno S, Nakai Y, Isayama $\mathrm{H}$, Yanai $\mathrm{A}$, Tahagara N, Miyabayashi K, Yamamoto K, Kawakubo K, Mohri D, Kogure H, Sakaki T, Yamamoto N, Sahahira N, Hirano K, Tsujino T, Ljichi H, Takeishi K, Akanuma M, Taka M, Koike K: Risk factors and early signs of pancreatic cancer in diabetes: screening strategy based on diabetes onset age. J Gastroenterol 2013;48:238-246.

15 Pleskow DK, Berger HJ, Gyves J, Allen E, McLean A, Podolsky DK: Evaluation of a serologic marker, CA19-9, in the diagnosis of pancreatic cancer. Ann Intern Med 1989;110: 704-709.

16 Marrelli D, Caruso S, Pedrazzani C, Neri A, Fernandes E, Marini M, Pinto E, Roviello F: CA19-9 serum levels in obstructive jaundice: clinical value in benign and malignant conditions. Am J Surg 2009;198:333-339.

17 Glenn J, Steinberg WM, Kurtzman SH, Steinberg SM, Sindelar WF: Evaluation of the utility of a radioimmunoassay for serum CA19-9 levels in patients before and after treatment of carcinoma of the pancreas. J Clin Oncol 1988; 6:462-468.

18 Nakaizumi A, Tatsuta M, Uehara H, Iishi $H$, Yamamura H, Okuda S, Kitamura T: A prospective trial of early detection of pancreatic cancer by ultrasonographic examination combined with measurement of serum elastase 1. Cancer 1992;69:936-940.

19 Tanaka S, Nakao M, Ioka T, Takakura R, Takano Y, Tsukuma H, Uehara H, Suzuki R, Fukuda J: Slight dilatation of the main pancreatic duct and presence of pancreatic cysts as predictive signs of pancreatic cancer: a prospective study. Radiology 2010;254:965-972.

20 Sakamoto H, Kitano M, Komaki T, Noda K, Chikugo T, Kudo M: Small cell carcinoma of the pancreas: role of EUS-FNA and subsequent effective chemotherapy using carboplatin and etoposide. J Gastroenterol 2009;44: 432-438.

21 Sakamoto H, Kitano M, Komaki T, Noda K, Chikugo T, Dote K, Takeyama Y, Das K, Ya- mao K, Kudo M: Prospective comparative study of the EUS guided 25-gauge FNA needle with the 19-gauge Trucut needle and 22-gauge FNA needle in patients with solid pancreatic masses. J Gastroenterol Hepatol 2008;55: 1785-1788.

22 Hanada K, Okazaki A, Hirano N, Izumi Y, Minami T, Ikemoto J, Kanemitsu K, Hino F: Effective screening for early diagnosis of pancreatic cancer. Best Pract Res Clin Gastroenterol 2015;29:929-939.

23 Egawa S, Toma H, Ohigashi H, Okusaka T, Nakao A, Hatori T, Maguchi H, Yanagisawa A, Tanakan M: Japan Pancreatic Cancer Registry; 30th year anniversary: Japan Pancreas Society. Pancreas 2012;41:985-992.

24 Chiang KC, Yeh CN, Lee WC, Jan YY, Hwang TL: Prognostic analysis of patients with pancreatic head adenocarcinoma less than $2 \mathrm{~cm}$ undergoing resection. World J Gastroenterol 2009;15:4305-4310.

25 Homma T, Tsuchiya R: The study of the mass screening of persons without symptoms and of the screening of outpatients with gastrointestinal complains or icterus for pancreatic cancer in Japan, using CA19-9 and elastase-1 or ultrasonography. Int J Pancreatol 1991;9: 119-124.

26 Lopez Hänninen E, Amthauer H, Hosten N, Ricke J, Böhmig M, Langrehr J, Hintze R, Neuhaus P, Wiedenmann B, Rosewicz S, Felix $\mathrm{R}$ : Prospective evaluation of pancreatic tumors: accuracy of MR imaging with MR cholangiopancreatography and MR angiography. Radiology 2002;224:34-41.

27 Adamek HE, Albert J, Breer H, Weitz M, Schilling D, Riemann JF: Pancreatic cancer detection with magnetic resonance cholangiopancreatography and endoscopic retrograde cholangiopancreatography: a prospective controlled study. Lancet 2000;356:190-193.

28 Holzapfel K, Reiser-Erkan C, Fingerle AA, Erkan M, Eiber MJ, Rummeny EJ, Friess H, Kleeff J, Gaa J: Comparison of diffusionweighted MR imaging and multidetector-row $\mathrm{CT}$ in the detection of liver metastases in patients operated for pancreatic cancer. Abdom Imaging 2011:36:179-184. 